\title{
El debate contemporáneo del Trabajo Social argentino: crítica al pensamiento sistémico
}

\author{
Contemporary discussion of the Argentine \\ Social Work: critics to systemic thinking
}

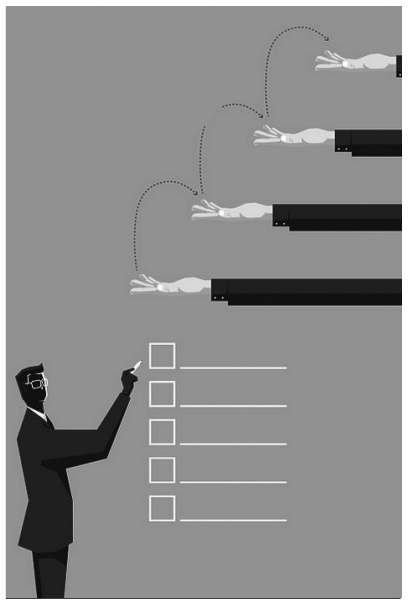

Sergio Daniel Gianna*

Resumen: El artículo presenta algunas de las reflexiones realizadas en la tesis doctoral acerca de la incidencia de la racionalidad formal-abstracta y el irracionalismo en el Trabajo Social argentino. Para ello, se analiza como la Teoría General de los Sistemas se constituye en una nueva expresión de una razón abstractiva y manipulatoria y como a partir de ella, se produce un corpus teórico y reflexivo dentro del Trabajo Social. Así, se estudian las principales consideraciones que dichos autores efectúan en torno a la intervención profesional y como éstas conducen a un nuevo Trabajo Social de carácter conservador.

Palabras-clave: Racionalidad formal-abstracta. Funcionalismo. Teoría General de los Sistemas. Trabajo Social. Intervención profesional.

\begin{abstract}
This paper displays some of the reflections carried out on the $\mathrm{PhD}$ thesis concerning the incidence of both the formal-abstract rationality and irrationalism within the Argentine Social Work. For this purpose, it is discussed how the Systems General Theory becomes a new expression of an abstractive and manipulating reason as well as how, therein, a reflexive and theoretical corpus takes place within Social Work. Thus, the discussion focuses on the major considerations made by such authors as regards the professional intervention leading to a new conservative-type Social Work.

Keywords: Formal-abstract rationality. Functionalism. Systems General Theory. Social Work. Professional Intervention.

* Licenciado en Trabajo Social, Escuela de Trabajo Social, Universidad Nacional de Córdoba. Magister en Trabajo Social, Facultad de Trabajo Social, Universidad Nacional de la Plata, Buenos Aires, Argentina. Doctorando en Ciencias Sociales, Facultad de Ciencias Sociales, Universidad de Buenos Aires. E-mail: sdgianna@gmail.com.
\end{abstract}




\section{Introducción}

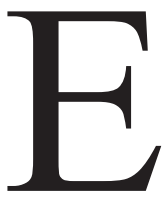

1 presente artículo tiene por finalidad el estudio acerca del pensamiento sistémico en el Trabajo Social argentino y sus implicancias para la intervención profesional de los trabajadores sociales.

Así, el trabajo se estructura a partir de dos momentos interrelacionados entre sí. En un primer momento, se abordan algunos elementos generales en torno a la racionalidad formal-abstracta, su carácter esencialmente manipulatorio y como existe entre el positivismo y el funcionalismo de Parsons una línea de continuidad con la Teoría General de los Sistemas.

Mientras que en un segundo momento, se analizan las principales implicancias teóricas, éticas y políticas de la perspectiva sistémica en Trabajo Social y cuales son sus puntos problemáticos en lo que hace al modo de desarrollar una intervención profesional desde la Teoría General de los Sistemas.

\section{La Teoría General de los Sistemas como expresión de la racionalidad formal-abstracta}

Lukács (1959) resalta que existen al menos dos fases en el pensamiento filosófico de la burguesía que se articulan con las determinaciones socio-históricas y el desarrollo de la lucha de clases. El primero de estos momentos se ubica desde el enfrentamiento de la burguesía frente al absolutismo feudal, ya que a partir del siglo XVI, y durante los dos siglos subsiguientes, se produce la transición entre el modo de producción feudal hacia el modo de producción capitalista. En la lucha contra el absolutismo feudal, la burguesía se constituyó en una clase ascendente, representando el "interés general del pueblo" (Marx, 1965) y consolidando una transformación no sólo en el plano de lo económico y lo social, sino también en lo cultural. Según Lukács, esta situación histórica hace que la ciencia y la filosofía tuvieran como preocupación el estudio de las "[...] leyes del proceso histórico, del progreso histórico-social, de descubrir y reducir a conceptos la razón en la historia y, sobretodo, la razón inmanente a la historia humana, la razón implícita en el propio movimiento de la historia en su conjunto" (Lukács, 1959, p. 100). 
Esto implica que en la fase ascendente de la burguesía existía una mediación profunda entre ciencia y concepción de mundo, ya que "[...] en la etapa de ascenso [es] una relación de fomento mutuo, en la línea descendente se traducen un entorpecimiento de una y otra" (Lukács, 1959, p. 86). ${ }^{1}$

Esta cuestión se encuentra vinculada a las contradicciones inherentes del proyecto societario impulsado por la burguesía, ya que, si por un lado, es un modo de producción revolucionario que permanentemente amplía las bases de reproducción mediante la creación de riqueza social, por el otro, el acceso a los productos del trabajo total de la sociedad es de carácter privado. Será con la "primavera de los pueblos", el alzamiento de la clase trabajadora en distintos países de Europa en 1848, cuando esta contradicción comienza a ser visualizada y la burguesía se convierte de clase progresista en clase dominante, actuando con el fin de defender el status quo alcanzado por la misma.

Es ese nuevo escenario societario introducirá significativas transformaciones en la función social que desempeña la filosofía y la ciencia, ya que se produce un corte cultural en la teoría social, desapareciendo del horizonte de la ciencia las bases fundamentales para la elaboración de una teoría social unitaria y totalizante: en primer término, el pensamiento clásico, tanto la economía política como la filosofía de Hegel, tomaban al trabajo como la actividad fundante del ser social destinada a la producción de valores y riqueza social. En segundo lugar, si se concibe a la realidad como algo racional y contradictorio, que se desarrolla bajo leyes objetivas que pueden ser aprendidas, esto significa que la sociabilidad capitalista es de carácter histórica y que puede ser superada por los hombres.

Si la razón en la fase ascendente de la burguesía expresaba la capacidad humana de aprender las determinaciones presentes en la realidad objetiva en sí, en la fase de decadencia la razón comienza a ser negada o se limita su papel en el rol de transformación de la naturaleza y la sociedad, ya que se

1. Para Lukács, será Hegel quien sintetice en su filosofía la concepción de mundo de la burguesía en su fase ascendente. Aquí no se dispone de espacio para su desarrollo, cabe destacar el papel de dicho filósofo en el descubrimiento de la categoría trabajo como actividad fundante del ser social, los aspectos ligados al método dialéctico, a la cuestión de la esencia y el fenómeno, entre otros. Sobre esto, ver Lukács (1970), Coutinho (1973), Gianna (2014). 
[...] renuncia a la ambición de proporcionar la respuesta a las últimas cuestiones del espíritu. En lo referente a la teoría del conocimiento esta tendencia se manifiesta por el agnosticismo que pretende que nada podemos saber de la esencia verdadera del mundo y de la realidad y que este conocimiento carecería, además, de utilidad alguna para nosotros. Debemos preocuparnos sólo de las conquistas de las ciencias, especializadas y separadas entre sí, conocimientos indispensables desde el punto de vista de la vida práctica de todos los días (Lukács, 1975, p. 23).

Es decir, la consecuente conformación de distintas ciencias especializadas, y la ruptura con la perspectiva de totalidad, no niega la producción de conocimiento científico, no obstante, éste tiene la particularidad de que está destinado a manipular ciertos dominios y porciones de la realidad, sin extraer de él generalizaciones y la construcción de una ontología o concepción del mundo. En relación a esto, Lukács afirma que las ciencias "[...] se hallan, con frecuencia, en condiciones de resolver directamente determinados problemas que la vida plantea, no pocas veces sin preocuparse gran cosa de las consecuencias filosóficas que de ello pueden derivarse [...]" (1959, p. 82).

En ese sentido Coutinho afirma que la manipulación habilita un dominio inmediato sobre el medio, mientras que la racionalidad es una apropiación de la objetividad natural o social y sus determinaciones presentes. Así, “[...] la manipulación impide no sólo una aprehensión rica y claramente determinada de la objetividad, sino también una correcta conciencia del significado humano y social de la praxis" (Coutinho, 1973, p. 70). ${ }^{2}$

La manipulación, que se extiende en la sociabilidad capitalista junto a una lógica instrumental y burocratizante, se expresa en la ciencia en la racionalidad formal-abstracta, que niega la contradictoriedad del objeto y afirma su equilibrio y progreso lineal, ya que, según Coutinho (1973), ésta se basa en el cálculo y

2. Cabe destacar que la manipulación no es per se algo negativo para el individuo y la sociabilidad, ya que ésta se liga a la vida cotidiana. En ésta, ciertos elementos de la práctica, que requieren inicialmente de una aprehensión de la realidad y un esfuerzo conciente del individuo para actuar sobre la misma, se vuelve algo no conciente, una nueva inmediatez o reflejo condicionado, que no requiere del individuo esfuerzo alguno para su consecución. En otras palabras, la manipulación permite a los individuos actuar sobre el medio y la realidad sin conocer las causas de la misma, por ejemplo, cuando uno enciende una luz uno sabe que mediante su interruptor es posible apagar o prender la luz, pero el mecanismo bajo el cual este funciona permanece desconocido para el sujeto. Sobre esto, ver Lukács (1966). 
las manipulaciones homogeneizadoras. Así, la reducción a reglas formales de la razón lleva a una ruptura con la perspectiva de totalidad, que queda limitada a un conjunto de datos y fenómenos que son analizados desde reglas formales establecidas por el intelecto.

De este modo, se rompe con una perspectiva de totalidad que reconoce a los hechos y fenómenos como parte de una totalidad más amplia que las contiene, cuyo movimiento, en su esencia, es de contradicción. Ya Hegel señalaba que la razón se basaba en un principio de identidad y de no identidad, es decir, de un movimiento de unidad de contrarios que establece momentos de síntesis y superación que se encuentra en un permanente devenir. En contraposición, se plantea la desaparición de la contradicción y si la existencia de ambigüedades, resueltas por el irracionalismo y la racionalidad formal-abstracta de modo complementario. Finalmente la realidad es tal cual la que se le presenta a los ojos del investigador, reconociendo sólo la superficie fenoménica de los hechos. Es decir, esta queda ligada al nivel de lo inmediato, epidérmico, y no alcanza la reproducción de la esencia. Así, se "[...] destierran de la realidad [...] los problemas de contenido, los problemas de la contradicción [...] y formaliza[n] hasta el extremo su objeto [y] destierran de sus preocupaciones cualquiera referencia a la objetividad de las contradicciones en el capitalismo" (Coutinho, 1973, p. 39).

En esta racionalidad formal-abstracta se expresa un uso práctico de los sujetos, ya que en ella “[...] el cómo necesariamente pasa a primer plano” (Lukács, 2012, p. 90). No obstante, junto a la racionalidad manipulatoria aparece una corriente irracionalista, que pone el acento en una dimensión subjetiva - emparentada con lo que Lukács (1981) llamó la "critica romántica del capitalismo" —, ya que mediante la misma, y por las vivencias personales, es posible alcanzar la realidad auténtica, disolviéndose el objeto - y con él su racionalidad inmanente - en una mera intuición subjetiva. Esta intuición es la resultante de la separación entre objetividad y subjetividad, entre intelecto y razón. ${ }^{3}$

Así, existe una unidad entre la racionalidad formal-abstracta y el irracionalismo, cuyo enlace se encuentra en el agnosticismo, ya que el primero decla-

3. Aquí apenas se hacen algunas alusiones al irracionalismo. Sobre esto, ver Lukács (1959), Lukács (1975), Coutinho (1973). 
ra la imposibilidad de conocer determinados aspectos de la realidad y éstos se convierten en el punto de partida del irracionalismo, basado en una subjetividad desobjetivada, ya que existe una realidad más profunda que la realidad objetiva, que sólo puede ser aprendida mediante la intuición como organon de conocimiento. ${ }^{4}$ Coutinho observa que a partir de entonces comienza a predominar en la filosofía y en la ciencia tres pilares fundamentales:

[...] un individualismo exacerbado que niega el carácter social del hombre, o la afirmación de que el hombre es una "cosa"; ambas posiciones acarrean la negación del momento (relativamente) creador de la praxis humana. En lugar del historicismo surge una seudohistoricidad subjetivista y abstracta o una apología de la positividad, que transforman la historia real (el proceso de surgimiento de lo nuevo) en algo "superficial" o irracional. En lugar de la razón dialéctica, que afirma la cognoscibilidad de la esencia contradictoria de lo real, contemplamos el nacimiento de un irracionalismo fundado en la intuición arbitraria, o un profundo agnosticismo que es la consecuencia de haber limitado la racionalidad a sus formas, puramente intelectivas (Coutinho, 1973, p. 23).

En ese sentido, la Teoría General de los Sistemas (de aquí en más TGS) se constituye en una expresión de esta racionalidad formal-abstracta manipulatoria, cuyos fundamentos teóricos se encuentran en ciertas continuidades con el positivismo y el funcionalismo.

Ya en el funcionalismo clásico, cuyo mayor exponente es Parsons, se observa una analogía respecto a los sistemas orgánico-naturales y los sistemas sociales. Dicho aspecto, remite a los planteos de Durkheim, quien señala, como claro exponente del positivismo clásico, que "[...] los fenómenos sociales son cosas y deben ser tratados como cosas" (Durkheim, 2002, p. 67) y son "[...] un orden de hechos que presentan características muy especiales: consisten en modos de actuar, pensar, de sentir, exteriores al individuo" (Idem, p. 40). Esto significa que existe una homologación e identidad entre los fenómenos sociales

4. Cabe destacar, que la adhesión al irracionalismo o la racionalidad formal-abstracta no siempre es el resultado de una adhesión conciente a los intereses de la burguesía, sino que se vincula con la imposibilidad de superar aquellos aspectos inmediatos y fenoménicos de la sociabilidad capitalista, ya que éstos se vuelven, en su apariencia inmediata, relaciones cosificadas y fetichizadas. 
con los fenómenos naturales, quitándole cualquier tipo de particularidad que pueda existir en la legalidad natural y en la legalidad social. Como afirma Coutinho (1973), Durkheim veda lo específico de la praxis humana y termina equiparándola con los objetos naturales manipulables, ya que los objetos son cosas "[...] exteriores, superiores y anteriores a los hombres" (Durkheim, 2002, p. 53) sobre las cuales los hombres no pueden actuar y modificar.

Para el positivismo el conocimiento científico debe estar dirigido a alcanzar ciertas regularidades y leyes que rigen la sociabilidad humana y sobre las cuales el hombre no puede actuar ni torcer su rumbo. Para Durkheim "[...] los fenómenos psíquicos y sociales son hechos como los otros, sometidos a leyes que la voluntad humana no puede desarreglar a su manera y que, por consiguiente, las revoluciones en el sentido propio de la palabra son cosas tan imposibles como los milagros" (Durkheim in Löwy, 1991, p. 25).

Así, “[...] la sociedad no es una simple suma de individuos, sino que el sistema formados por su asociación representa una realidad específica que tiene caracteres propios" (Durkheim, 2002, p. 159). Estos caracteres del sistema, distintos al de sus partes, expresan alguna de las ideas que luego la TGS retomará. En otro de los pasajes de Las reglas del método sociológico, Durkheim profundiza esto diciendo: "[...] un todo no es idéntico a la suma de sus partes, es otra cosa cuyas propiedades difieren de las que presentan las partes que lo componen" (Idem, p. 158).

No obstante, si la sociedad es diferente de las partes que la constituye, esto no significa que sean un todo indiferenciado, sino que, por el contrario, éstas "Están constituidas, no por una repetición de segmentos similares y homogéneos, sino por un sistema de órganos diferentes, cada uno con su función especial y formados, ellos mismos, de partes diferenciadas" (Durkheim, 1987, p. 216) y estos "No se encuentran ni yuxtapuestos linealmente, como los anillos de un anélido, ni encajados unos en otros, sino coordinados y subordinados unos a otros [...]" (Idem, p. 216) y si uno de éstos ocupa “[...] una situación particular y si se quiere privilegiada; es debida a la naturaleza del papel que desempeña y no a una causa extraña a esas funciones, a una fuerza cualquiera que se le comunica desde fuera" (Idem, p. 216).

Aquí queda en evidencia que la sociedad, desde la óptica de Durkheim, es un sistema social u organismo con órganos que cumplen funciones diferencia- 
das. De ello, se infiere, por un lado, que los privilegios de ciertos órganos respecto a otros es una cuestión completamente natural, debido a la función que cumplen unos y otros, $\mathrm{y}$, por otro, que si un órgano quisiera cumplimentar otro rol o función traería un desequilibrio y anomia en el conjunto del organismo.

Esta tradición positivista adquiere continuidad, aunque con algunas particularidades que la distinguen de Durkheim, por Parsons, quien propone desarrollar una Teoría General basada en hipótesis lógicas y abstracciones teóricas. Es decir, el funcionalismo de Parsons posee un alto nivel de abstracción, hecho que lo distingue de los empiristas norteamericanos que profundizaron las reglas sociológicas planteadas por Durkheim. La abstracción analítica tiene por finalidad la creación de ciertos conceptos que son separados del movimiento de la historia y universalizados para toda forma de sociabilidad. Para Gouldner "[...] la prueba de significación de estos conceptos no es que conduzcan a hipótesis o proposiciones susceptibles de ser puestas a prueba [...]" (Gouldner, 1973, p. 195), por el contrario, éstas alcanzan su cometido cuando '[...] logra[n] 'mostrar' que un conjunto de sus categorías o conceptos puede ser aplicado a diversos sectores sociales o a diferentes niveles de la vida social [...]” (Idem, p. 195).

Así, la preocupación del funcionalismo clásico de Parsons se vincula a la construcción de modelos acerca de la acción social, a partir de variables e indicadores aplicables a cualquier momento histórico. Aquí se observa dos aspectos fundamentales de la racionalidad formal-abstracta: por un lado, la eliminación de la historia, ya que la finalidad de Parsons conduce a la construcción de ideas ahistóricas aplicables a cualquier momento histórico, y, por otro, esto se complementa con la elaboración de modelos abstractos y formales que eliminan los problemas de contenido.

Según Santos de Souza (2001), Parsons tiene como punto de partida de su análisis teórico a la acción social, la cual, posee distintos elementos que la componen, constituyendo una totalidad compleja o un sistema de acción. Esto expresa una concepción sistémica, en la que el sistema social es abierto e interactúa con el sistema cultural y el de la personalidad, así como el del medio ambiente físico. La reciprocidad entre estos elementos conducen a "[...] un molde [...] y testimonia una estabilidad en el tiempo [...] [lo que] implica también una noción de estabilidad, algo que tiende a mantenerse (mantener la estructura y los procesos) [...]" (Santos de Souza, 2001, p. 10-11). 
La estabilidad en los sistemas lleva a Parsons a concebir dos aspectos fundamentales: la existencia de una estructura ligada a los elementos que le otorgan relativa estabilidad y ciertas funciones que deben cumplirse. Toda interacción se encuentra organizada por un sistema de papeles que, al mismo tiempo, se estructuran en colectividades interrelacionadas. Este planteo destaca como lo normativo cumple una función central en el pensamiento de Parsons, ya que éste sirve como "[...] criterio normativo para la elección entre alternativas de orientación que están intrínsecamente abiertas en una situación [...]" (Parsons, 1999, p. 44). Así, toda acción social es "normativamente orientada" y

El problema del orden, y por ello de la naturaleza de la integración de los sistemas estables de la interacción social (o lo que es igual, de la estructura social), se centra en la integración de la motivación de los actores con los criterios normativos culturales que integran el sistema de acción, en nuestro contexto interpersonalmente. Estos criterios son [...] pautas de orientación de valor, y como tales son una parte particularmente crucial de la tradición cultural del sistema social (Parsons, 1999, p. 27).

Este extracto permite inferir que una sociedad se encuentra ordenada, y en un relativo equilibrio, cuando los individuos comparten entre sí un conjunto de normas culturales y cumplen ciertos papeles dentro de ésta. Según Robertston (1970), el sistema cultural brinda el contenido de los valores y los papeles que los sujetos desempeñan depende del sistema de la personalidad. Mediante ello, es posible alcanzar una tendencia al equilibrio, tanto mediante una institucionalización, que articula las actividades sociales con los valores dominantes, y la interiorización, que alude a la incorporación de la cultura en la personalidad del individuo.

Esta tendencia, a la "[...] entera moralización de las teorías sociales abarcativas" (Netto, 1997, p. 42), es lo que conducirá luego a la TGS a concebir que los problemas sociales se encuentran en sus aspectos relacionales y en las gratificaciones que los individuos y grupos sociales obtienen mediante sus intercambios, que deben reconocer sus posibilidades y límites a partir del sistema social, el sistema cultural y la personalidad. Como el sistema social tiende a 
lograr una relativa estabilidad, esta depende de que las partes admitan cuál es su lugar y la función que deben desempeñar dentro de la misma.

Será Ludwig Von Bertalanffy, biólogo de origen alemán, quien sintetice en su obra La teoría general de los sistemas las principales proposiciones del pensamiento sistémico. En dicha obra, donde se compilan diversas intervenciones y artículos del autor producidas desde 1945, aparece con suma claridad una misma concepción de totalidad basada en una concepción organística, que señala que

Es necesario estudiar no sólo partes y procesos aislados, sino también resolver los problemas decisivos hallados en la organización y el orden que los unifican, resultantes de la interacción dinámica de partes y que hacen el diferente comportamiento de éstas cuando se estudian aisladas o dentro del todo (Bertalanffy, 1980, p. 31).

Aquí se traslucen dos elementos de continuidad entre el funcionalismo clásico y el "nuevo funcionalismo": por un lado, que para la concepción sistémica las partes sólo existen en función de la totalidad, no siendo éstas totalidades de mayor o menor complejidad que guardan una autonomía relativa, $\mathrm{y}$, por otro, como la TGS va a plantear las leyes generales para cualquier sistema - sea éste social, natural o de otra índole, o abierto o cerrado (todas características que se irán retomando en la exposición) - y se afirma que es posible conocer todas las partes constitutivas de un sistema.

Ambos elementos adquieren concreción en el marco de una visión teórica plenamente formalista que, en primer término, afirma que la TGS es aplicable a cualquier tipo de sistema, siendo un nuevo paradigma científico que "[...] es circunscribible como "ciencia de los sistemas", o sea la exploración y la explicación científicas de los "sistemas" de las varias ciencias (física, biología, psicología, ciencias sociales ), con la teoría general de los sistemas como doctrina de principios aplicables a todos los sistemas [...]" (Bertalanffy, 1980, p. XIII). En cuanto nuevo paradigma científico, la teoría sistémica apunta a alcanzar un conjunto de proposiciones teóricas posibles de aplicarse a sistemas de distinta naturaleza, ya que "[...] existen modelos, principios y leyes aplicables a sistemas generalizados o a sus subclases, sin importar su particular género, la 
naturaleza de sus elementos componentes y las relaciones o "fuerzas" que imperen entre ellos" (Bertalanffy, 1980, p. 32).

Según Bertalanffy (1980) no existirían distinciones fundamentales entre los distintos tipos de sistema, con lo que el sistema de la naturaleza no tendría una legalidad distintiva a la de la sociabilidad humana. Estas afirmaciones del autor expresan ciertos puntos de contacto con los planteos de Durkheim acerca del organicismo y la concepción de la sociedad como un organismo compuesto de órganos entrelazados entre sí, donde las leyes que puede construir las ciencias sociales se asemejan a las que producen las ciencias naturales. Esta indiferenciación de los sistemas significa que

Podemos [...] buscar principios aplicables a sistemas en general, sin importar que sean de naturaleza física, biológica o sociológica. Si planteamos esto y definimos bien al sistema, hallaremos que existen modelos, principios y leyes que se aplican a sistemas generalizados, sin importar su particular género, elementos y "fuerzas" participantes (Bertalanffy, 1980, p. 33).

De este modo, el tipo de leyes que produce la TGS es una abstracción generalizable de aspectos coagulados del movimiento de la realidad, cuyo elemento central es el estadístico, que borra las particularidades y demuestra cuantitativamente una misma tendencia. Estas leyes y modelos teóricos son posibles de configurarse mediante la matematización de la realidad, ya que “[...] la teoría general de los sistemas es un ciencia general de la "totalidad" [...] En forma elaborada sería una disciplina lógico-matemática, puramente formal en sí misma pero aplicable a las varias ciencias empíricas" (Bertalanffy, 1980, p. 37), promoviendo un nuevo paradigma científico que produzca el pasaje de la "[...] teoría de las categorías [a] [...] un sistema exacto de leyes lógico-matemáticas” (Bertalanffy, 1980, p. 88).

Lukács (2012) va a recalcar que la tendencia al formalismo, propia de la racionalidad formal-abstracta, oculta que toda determinación cuantitativa es una cantidad de "alguna cosa", de un substrato categorial que son los presupuestos de las relaciones cuantitativas reales. Así, se descifran los fenómenos a partir del medio homogéneo de la matemática y se establece una unidad absoluta de la ciencia que excluye las diferencias. Según el autor 
El neopositivismo, como vimos, desea resolver esa cuestión por la exclusión de toda ontología, por la simple unidad del "lenguaje" científico y por su tipo de manipulación logicista. De ese modo, todas las formas específicas de ser pierden forzosamente su particularidad interna, debiendo ser tratadas según el modelo — conforme la interpretación neopositivista- de la física moderna (Lukács, 2012, p. 69).

Los señalamientos de Lukács (2012) reflejan como el neopositivismo, y la TGS al retomar dichos planteos, tiende a constituir una totalidad formal que, aplicando la lógica de las matemáticas y la física, puede alcanzar una concepción globalizadora de la realidad. Pero, esto conduce, al hacerlo desde una racionalidad formal-abstracta, a desempeñar una función social claramente apologética, ya que buscan "[...] transferir los problemas y las contradicciones de la vida real [...] a la esfera legítima de la razón formalmente omnipotente, "transcendiendo" así, idealmente, en términos de los postulados formales universalmente válidos, la conflictividad real [...]" (Mészáros, 2011, p. 30).

Lukács (1959) observa que con el surgimiento del irracionalismo y la racionalidad formal-abstracta imperan dos tipos de apologéticas, cuya función es presentar en el plano del pensamiento científico una justificación y naturalización del estado actual de las cosas. Según el autor, existe una apologética directa que niega la existencia de contradicciones y conflictos, mientras que la apologética indirecta exhibe los aspectos históricos, y los conflictos y determinaciones históricas de un período dado, como "[...] cualidades inherentes a la existencia humana en general, a la vida misma, sin más" (Lukács, 1959, p. 167).

En el caso de la TGS se observa una apologética indirecta ya que, de forma general, se refiere a que si hay desajustes en un sistema este tiende siempre al equilibrio. Este proceso es caracterizado por Bertalanffy al decir que "En el estado de totalidad, una perturbación del sistema conduce a la introducción de un nuevo estado de equilibrio" (Bertalanffy, 1980, p. 71). Pese a la distinción entre los sistemas cerrados y los abiertos -ya que en el primero al no tener un intercambio con el medio supone que el estado final está determinado a partir de las condiciones iniciales y en el segundo al haber inputs y outputs distintivos hace que puedan "[...] alcanzarse el mismo estado final partiendo de diferentes condiciones iniciales y por diferentes caminos” (Idem, p. 40) - existiría una tendencia a un mantenimiento homeostático, esto es, a un equilibrio. 
Estos elementos generales de la $\mathrm{TGS}^{5}$ tendrán una incidencia significativa dentro del Trabajo Social, sobretodo, en lo que respecta al tipo de intervención que los profesionales deberían llevar a cabo, ya que si se piensa en el sistema social se podría afirmar que su dinámica natural se inclina al equilibrio y que, a lo sumo, debe actuarse sobre aquellas partes que rompen con dicha dinámica, de modo de reestablecer los lazos sociales existentes y promover su carácter homeostático. A continuación se buscará develar como esto se presenta en el Trabajo Social, sus implicancias para la intervención profesional y como en ella irrumpe con suma claridad una apologética indirecta.

\section{Trabajo Social e intervención profesional desde un enfoque sistémico}

Antes de iniciar con las observaciones críticas en torno a la incidencia de la TGS en la intervención profesional de los trabajadores sociales, cabe comenzar por retomar algunos elementos en torno a como esta perspectiva concibe la génesis del Trabajo Social. Éste es considerado como un servicio dirigido a “[...] brindar servicios a la población marginal y alienada" (Chadi, 2005, p. 19). Así, "Estos comienzos están emparentados con las buenas intenciones de personas colocadas socialmente en mejores condiciones personales para brindar "ayuda" a los seres más necesitados, en principio respecto de sus economías" (Chadi, 2005, p. 19). Según Chadi, estas acciones empiezan a sistematizarse y la beneficencia se reguló dando lugar a instituciones encargadas de encarar dichas acciones. Este origen del Trabajo Social estaría emparentado al asistencialismo, comprendido como el "[...] servir en algunas cosas o tareas a otros" (Chadi, 2005, p. 21).

Estas referencias claramente plantean al Trabajo Social como el resultante de una evolución de las formas de ayuda y caridad, siendo una continuidad de la tesis endogenista que explica el surgimiento de la profesión. En la misma,

5. Cabe destacar que también se encuentran en Bertalanffy una cierta incidencia del irracionalismo, sobretodo, de Spengler, en lo que hace a vincular la "matematización" de la realidad con un "perspectivismo", ya que si existe un lenguaje homogeneizador de todos los sistemas como las matemáticas, su interpretación siempre depende de la perspectiva histórica desde el cual se haga, llevando a un relativismo absoluto acerca de las conclusiones teóricas del hacer científico. Sobre esto, ver Gianna (2014). 
predomina una visión de exterioridad entre la historia general y el Trabajo Social, ya que no se capta a la profesión inserta en el conjunto de las relaciones sociales, sino que se lo hace desde una óptica particularista, en la que ciertos individuos harían evolucionar y desarrollar las formas de ayuda hasta adquirir su forma actual con el Trabajo Social (Montaño, 1998).

Resulta interesante que dentro del pensamiento sistémico en Trabajo Social predomina una visión, en algún caso de manera explícita, de una nueva fase o etapa en la evolución del Trabajo Social ligada a lo que Chadi (2005) llama el Trabajo Social independiente. Comparando esta situación con el crecimiento de un individuo, la autora señala: "Todo ser humano inicia su existencia con un alto grado de dependencia [...]. A medida que progresa en sus etapas evolutivas, se acrecientan los grados de independencia, hasta transformarse en un ser autónomo" (Chadi, 2005, p. 28) y, con ello, se estaría en una nueva fase o momento asociado a la independencia y la autonomía.

Así, el Trabajo Social independiente, al encontrarse en una fase de aparición, oscila entre el temor que conduce a quedarse en las funciones y atribuciones dadas y un deseo de crecimiento e innovación. Según Chadi,

[...] existe una contradicción entre la demanda social y de los servicios institucionales, que asocian al Trabajador Social como solamente capacitado para intervenir en los niveles sociales marginales, cuando en realidad la formación e incumbencias son amplias y no sectarias, pudiendo dirigirse a cualquiera que pueda tener problemas sociales [...] (Chadi, 2005, p. 24).

Si bien se afirma que el Trabajo Social independiente no es incompatible con aquél que se desenvuelve en el marco de instituciones estatales y públicas, la posibilidad de esta nueva "condición laboral" de los trabajadores sociales se ligaría también con una evolución en la función social que la profesión desarrolla, pasando del asistencialismo anteriormente señalado, que coloca al usuario como sujeto pasivo y al profesional como activo, a la asistencia, que establece mayor simetría entre el profesional y los actores involucrados. Habría una suerte de “fusión” entre “[...] 'las identidades asistenciales y terapéuticas', que se entrelazan al tomar el término 'terapéutico', como común a lo psíquico y social” (Chadi, 2005, p. 30-31). Así, el Trabajo Social genera una intervención socio-tera- 
péutica o un Trabajo Social clínico, al encontrarse vinculado con "[...] procedimientos para el tratamiento que conduzcan a la reducción de los factores que originan el sufrimiento humano [...] [y] tiene como meta la mejora del mantenimiento psicosocial de los individuos, las familias y los grupos" (Idem, p. 22).

Con ello, la función social del Trabajo Social independiente se liga a “[...] la mejora y el mantenimiento del funcionamiento psicosocial de los individuos, la familia y los grupos [...]" (Chadi, 1997, p. 59), mediante un modelo de intervención "psico-relacional-social" en el que converge lo sistémico (relacional) y el Trabajo Social (el contexto). Así, el Trabajo Social independiente y sistémico propone como foco de intervención el pasaje de lo intrapsíquico a lo relacional, ya que este último amplía el campo de comprensión de la conducta humana (Chadi, 2005).

Estos extractos dan cuenta de cómo un sector del Trabajo Social construye un proyecto profesional ligado al ámbito privado y cuyo eje de atención se encuentra en dos aspectos interligados entre sí: el primero, un progresivo viraje en torno a la población usuaria que puede acceder al accionar profesional de los trabajadores sociales en el sector privado. El trabajador social monta un "consultorio social", desligado del Estado, al que asisten "clientes" que tengan la capacidad económica de pagar por la intervención profesional desarrollada por el trabajador social. En segundo término, diversos autores afirman mediante la recuperación de distintos documentos de la categoría profesional, como es el caso de la asamblea de Montreal de 1998, que el Trabajo Social se liga a "[...] la resolución de problemas en las relaciones humanas, el cambio social, el poder de las personas mediante el ejercicio de sus derechos y su liberación, y mejora de la sociedad [...]" (Chadi, 2005, p. 23) y luego señala que "Los principios de los Derechos Humanos y la Justicia Social son esenciales para el Trabajo Social" (Idem, p. 24). Sin embargo, si el Trabajo Social tiene vinculación con los derechos humanos (y sociales) y a la justicia social, estos, ¿Qué nivel de injerencia y vinculación pueden tener con un abordaje privado? Es decir, se pasa de una lógica de derechos a otra de carácter mercantil en el Trabajo Social privado e "independiente". Al mismo tiempo, aparece una visión reduccionista de los derechos sociales, los cuales, parecieran estar estrictamente ligados a los "pobres", mientras que los otros grupos y clases sociales deberían resolver sus problemas sociales de manera independiente y privada. 
Por otra parte, se propone que desde la intervención se deje de trabajar con "lo que falta" o lo disfuncional y se parta de los recursos y de los aspectos sociales de los individuos y grupos sociales. Según Díaz Usandivaras, el Trabajo Social

[...] ayuda a la gente que no logra por las suyas resolver sus problemas, descubriéndoles sus recursos y habilidades no utilizadas, ampliando alternativas, desbloqueando restricciones en las conductas, a veces consecuencia de creencias y maneras de pensar, que limitan su competencia (apud Chadi, 2005, p. 86-87).

La visión sistémica de la intervención profesional y su finalidad coloca el énfasis en la capacidad que poseen los individuos y a lo sumo los grupos sociales, para resolver sus problemáticas sociales mediante sus propios recursos. Esto conduce, por un lado, a introducir una "inducción comportamental", al ubicar los problemas sociales al nivel de lo relacional, como si este fuera el único aspecto que se presenta dentro de la intervención profesional y hubiera "relaciones válidas y otras inválidas" y, por otro lado, este énfasis excesivo en lo relacional termina ocultando y negando aquellas determinaciones macroestructurales que atraviesan a los procesos sociales y a las problemáticas, como es el caso de las mediaciones existentes entre el metabolismo del capital y sus consecuencias deshumanizantes y reificadas con las que se enfrenta el profesional en la intervención. Pese a que se advierte el pasaje de lo psicológico a lo relacional, también aparece una tendencia a la "psicologización de la cuestión social", al concebir, particularmente en la familia, determinadas etapas en el ciclo de una familia y "comportamientos esperables" en sus miembros. Según Chadi, en la intervención con familias es necesario "Investigar en que etapa del ciclo de la vida familiar se encuentra la familia y si las reglas de funcionamiento de la misma corresponden a dicho ciclo" (Chadi, 1997, p. 35).

Esta moralización de las relaciones sociales, y su tendencia a inducir comportamientos "normales" sobre los "anormales", es la resultante de individuos que tienen baja su autoestima. La misma tiende a naturalizar las relaciones sociales, ya que apunta a "[...] la propia valoración que se tiene de uno mismo, con la posibilidad de aceptarse y de respetarse. Se relaciona con la aptitud de afirmar las capacidades, habilidades y entender las limitaciones" (Calvo, 2009, 
p. 30). Traduciendo el contenido de este pasaje, se advierte que los individuos deben respetarse como "pobres", ya que esto se liga a sus capacidades y habilidades propias, aceptando sus limitaciones. Con ello, el trabajo de la autoestima no supone que el mismo esté acompañado de una mejora en las condiciones de vida de los individuos y el acceso a recursos de diversa naturaleza, sino que, por el contrario, esto significa trabajar con el "ser interior" de los individuos, ya que "[...] supone revisar el sistema de creencias para intentar que la familia se de cuenta si actitudes, modos de actuar, pensamientos que tienen sobre ellos mismos o ideas sobre determinados temas, son verdaderamente de ellos o no" (Calvo, 2009, p. 34).

Inclusive, el "origen de todos los males sociales" se relaciona con la escasa autoestima, ya que "El escaso amor a sí mismo es el origen de varios conflictos en los seres humanos. Dificultades en el trabajo, imposibilidad de concretar relaciones afectivas saludables, enfermedades, tienen su origen en un no poder aceptarse como se es [...]" (Calvo, 2009, p. 31). Por ejemplo, si existen huelgas obreras, demandas a los empleadores, éstas no plantearían reivindicaciones concretas frente a situaciones particulares, por el contrario, serían un conjunto de sujetos que al tener baja su autoestima demandan, exigen y reclaman por su propio descontento. Así, queda en evidencia no sólo la directa moralización del individuo, sino la reducción de toda conflictividad social a una dimensión motivacional vinculada a la insatisfacción. El rechazo a la conflictividad social es reconocida por la autora cuando afirma que "Dentro de las funciones del Trabajo Social, la 'promoción de la salud' nos insta a realizar todo lo necesario para evitar que los problemas y conflictos familiares y sociales se produzcan en nuestra sociedad" (Calvo, 2009, p. 21). ${ }^{6}$

No obstante, esta finalidad de la intervención profesional quedaría inconclusa si no se analizan las otras funciones que el Trabajo Social tiene como profesión. Para Reynoso y Calvo existe una de carácter preventiva ligada a “[...] la no aparición de enfermedades y conflictos sociales. Realiza una labor educativa, trasmite nociones de autocuidado y autoasistencia [...]" (Reynoso y Calvo, 2003, p. 24), otra curativa que apunta a "[...] colabora(r) asesorando a

6. Subyace en este pasaje, ligado a la "promoción de la salud", que los problemas sociales son "enfermedades", mientras que el equilibrio y la estabilidad un estado de salud social. 
las personas para que logren solucionar sus situaciones conflictivas" (Idem, p. 25), una constructiva que "[...] tiende a cooperar y colaborar para mejorar los recursos de la comunidad" $\mathrm{y}$, finalmente, una paliativa que "[...] intenta aliviar los estado de necesidad de las personas" (Idem, p. 25).

Estas funciones desempeñadas por los trabajadores sociales, al mismo tiempo, deben estar acompañadas por ciertas aptitudes ligadas a una “[...] cualidad personal: tener la capacidad de sentir frente a su entrevistado, identificarse con sus problemas, dejar de lado prejuicios, mandatos y creencias limitadoras" (Reynoso y Calvo, 2003, p. 19). Así como el Trabajo Social "[...] necesita de personas con respeto y admiración por la condición humana" (Chadi, 2005, p. 32). Es decir, los profesionales además de adquirir competencias técnicas deberían ser capaces de tener ciertos atributos como personas, siendo "[...] gente con un trascendente grado de vocación de entrega, y de dar y devolver lo que humildemente hemos tenido la suerte de aprender" (Idem, p. 27).

De este modo, la finalidad y las funciones de la intervención profesional de los trabajadores sociales se vincula al proceso reconstructivo que realizan de la realidad, advirtiendo, por un lado, ciertas particularidades en el escenario contemporáneo dadas por una crisis que es "[...] una situación compleja, difícil de transitar. En la cual se ponen en evidencia la presencia o ausencia de los soportes internos o externos de la persona o grupo social" (Reynoso y Calvo, 2003, p. 79). Este diagnóstico de época expresa como la intervención profesional del trabajador social debe estar dirigida a "[...] activar los recursos propios del cliente, suponiendo un desarrollo a través de su autodeterminación" (Chadi, 2005, p. 22). Esta concepción, centrada en el cliente, sus recursos y el autodesarrollo, es la resultante de una mirada puesta en lo relacional, que advierte que el hombre es un "tejido de relaciones" (Chadi, 1997) pero, entre las cuales, hay una que adquiere relevancia: la familia. En la misma, los individuos no sólo inician un proceso de socialización y de reglas interactivas que son internalizadas, sino que

Todo sistema dicta sus propias pautas de funcionamiento, que establecen una dinámica y su interacción. Estas reglas permanecen estables de acuerdo a cada ciclo evolutivo, es decir que la familia atraviesa un determinado período sin cambios trascendentes. Dicha característica determina su condición de homeos- 
tasis, cualidad que es entendida como un estado relativamente constante que se mantiene mediante su autorregulación (Chadi, 2000, p. 44).

Aquí se observa como la tendencia de la TGS aparece en la concepción de familia, la cual, pese a poder considerarla como un sistema abierto, significando que existe un intercambio de entrada (input) y salida (output) entre el sistema y el medio, ésta tiende a un equilibrio dado por su autorregulación. Así, se tensiona y polariza entre el cambio y la conservación lo que es un proceso dialéctico que incluye conjuntamente la conservación, la negación y la superación. No obstante, lo relevante de esta visión de la familia como sistema se vincula a la función social que le es atribuye a la misma:

La familia, como integrante de la Red primaria, es la unidad estructural mínima dentro del universo del tejido relacional. Esta cualidad es vital tenerla en cuenta ya que, en el interior de la misma, habitualmente se gesta la disfuncionalidad que luego dará lugar a la convocatoria de los demás circuitos intersistémicos que se convertirán en la "extensión de apoyo" para el encuentro de soluciones. Es decir que la problemática, como emergente de una disfuncionalidad general, se manifiesta como síntoma dentro de esta micro agrupación (Chadi, 1997, p. 40).

Este pasaje retrata como la familia es, para el abordaje sistémico, la unidad esencial, la estructura mínima, de todo sistema social. Esto explica porqué este enfoque teórico prioriza en la intervención profesional el trabajo con familias, ya que se concibe que en éstas se producen las disfuncionalidades que pueden extenderse a otros sistemas sociales o redes secundarias. En otras palabras, en la familia se generan problemas relacionales que pueden tener algún grado de impacto sobre la propia red primaria y sobre aquellos sistemas con el que mantienen vínculo (red secundaria e institucional). En ese sentido, hay una responsabilización de la familia acerca de los problemas sociales, ya que en el núcleo de ésta se generan ciertas problemáticas sociales. Por ello, el trabajador social debe activar "[...] dichas redes primarias, como búsqueda de cooperaciones 'naturales' que acrecienten la fe del sistema familiar en sí mismo y en sus posibilidades, aumentando como consecuencia directa su autoestima y su propia valorización" (Chadi, 1997, p. 46). 
La preocupación constante acerca de lo relacional, presente en la mayoría de las citaciones hechas hasta el momento, tiende a convertirla en una abstracción recortada de la totalidad social e introducir una visión de que ciertos vínculos, al ser desajustados del automantenimiento homeostático, deben ser adaptados al sistema social. Inclusive, esto se articula con las concepciones acerca de la red secundaria e institucional y el tipo de vinculación que éstas tienen con la familia. El primer tipo de red, el secundario, refiere a relaciones más próximas a la red primaria, como los grupos recreativos, religiosos y laborales que pueden actuar como "redes de contención" o "extensión de apoyo" frente a un desequilibrio familiar. Éste será el objetivo del trabajo de red que, partiendo de la familia, busca establecer "puentes" con las redes secundarias para "[...] instrumentar una organización coherente en la red social que garantice la salud en sentido general, a través de su transformación en una unidad de apoyo, fortalecimiento y esclarecimiento" (Chadi, 1997, p. 76). Así, la solución a los problemas sociales se encuentra en los recursos y capacidades propias de la familia o en las redes secundarias.

La aprensión de estos aspectos de la realidad da cuenta de porque la perspectiva sistémica obvia las determinaciones macroestructurales que atraviesan a las problemáticas sociales y a las relaciones entre los individuos. Pero, también, permite comprender la razón por la que se desdeña la lógica de derechos, ya que ésta generaría un cierto grado de dependencia de los individuos a sistemas considerados artificiales y no naturales. En palabras de Chadi, "La convocatoria del 'sistema profesional' suele generar una 'perturbación ecológica' [...] se accede a ellas, por necesidades específicas que las tramas primarias no pueden satisfacer" (Chadi, 1997, p. 57). Y, en otro de sus textos, la autora, citando a Díaz Usandivaras, completa estas ideas comparando el trabajo con la red primaria y lo que sucede con la inclusión de la red institucional, ya que en la primera se "[...] retiene la responsabilidad por la conducta y por sus resoluciones en el individuo y en su familia, apelando a sus propios recursos, en vez de tender a la desresponsabilización, por depositación de éstos en las instituciones, perpetuando la dependencia" (Chadi, 1997, p. 47).

Aquí, queda en evidencia que la TGS, y su mediación con la intervención profesional, es un planteo teórico que aborda los problemas sociales, ligados a la reproducción objetiva y subjetiva de los individuos, sin captar las mediaciones 
que la constituyen, al tiempo que refuerza la faz privada de la atención de la "cuestión social", al apelarse a los recursos y capacidades propias de los individuos. De igual modo, esta "psicologización de la cuestión social” es acompañada por una tendencia al moralismo alienante que transforma la función social de la moral, pasando ésta de ser una instancia mediadora entre el individuo particular y el género humano a convertirse en una trasmisora y reproductora de las normas que se vuelven costumbres en la vida cotidiana. Para Barroco, "En la sociedad de clases, la moral cumple una función ideológica precisa: contribuye para una integración social que viabiliza necesidades privadas, ajenas y extrañas a las capacidades emancipadoras del hombre" (Barroco, 2004, p. 62).

Respecto a esto, se advierte que la fuerte moralización e individuación de los problemas sociales conducen a una familiarización de la intervención profesional, como si el enfoque sistémico fueran la expresión teórica de la orientación que adoptan las políticas sociales en los últimos años, no sólo con una focalización en las familias, sino también en el rol de las mujeres y la maternalización de las responsabilidades y deberes ligadas a las políticas sociales y el acceso a los recursos y servicios no mediados por el mercado de modo directo. Al respecto, Mallardi alude que

Mediante ésta, la estrategia moralizante se extiende a los seres sociales que la componen, haciéndolos responsable de lo que le sucede a cada uno de sus miembros. Así, la estrategia de individualización de la "cuestión social" pasa a necesitar de la funcionalidad de la familia para garantizar su finalidad. Por ello, tanto en la retórica como en la práctica, la familia pasa a constituirse en el fundamento y horizonte de todas las estrategias que intervienen en la "cuestión social" (Mallardi, 2013: 43).

De este modo, la moralización y "psicologización de la cuestión social" de los individuos y familias (la red primaria) son los contenidos que subyacen en la finalidad de la intervención profesional, sobretodo, al focalizar en los aspectos relacionales y en los puentes que los mismos deben crear con la red secundaria e institucional.

Finalmente, cabe destacar que existe dentro de esta perspectiva teórica una tendencia al metodologismo propio de la racionalidad formal-abstracta, aunque 
cabe destacar que las “[...] fases están ligadas una con otra y requieren de una conducta que permita "diagnosticar, evaluar e intervenir" permanentemente [...]" (Chadi, 1997, p. 91). Pese a esta advertencia, se proponen pautas metodológicas generales diferenciadas por los sujetos de la intervención, sea por el caso individual, familiar, grupal o comunitario.

Respecto al vínculo con los sujetos de la intervención, sean individual-familiares; grupales o comunitarios, se requiere de "[...] etapas de inclusión; de intervención propiamente dicha, y por último de desinvolucración" (Chadi, 2005 , p. 63), esto es, un acercamiento progresivo para conocer los problemas sociales, luego intervenir sobre los mismos y finalmente dar un cierre al accionar profesional al ser un sistema no ecológico que sólo actúa en situaciones puntuales y necesarias.

Junto a estos momentos, que orientan el vínculo con los sujetos de la intervención, se propone una serie de fases para intervenir sobre las problemáticas sociales. Calvo recalca que la metodología requiere de una serie de fases o etapas ligadas al

Estudio de la situación de la familia que se acerca, sus dificultades y la demanda. El estudio del medio familiar y de la red social [...] La elaboración y armado del genosociograma. El diagnostico social o evaluación diagnóstica. La intervención socioterapéutica o tratamiento social. Trabajo de red (Calvo, 2009, p. 24).

En síntesis, la TGS en el Trabajo Social conduce a un abordaje profesional de un ámbito "coagulado" de la realidad, la red primaria o la familia, en la que se encontraría la génesis de los problemas sociales, ya que los mismos serían producto de cuestiones vinculares, relacionales y de autoestima. Es por ello, que el trabajador social debe actuar sobre ellos, mediante un conjunto de procedimientos metodológicos, para fortalecer la red primaria.

\section{Consideraciones finales}

Como se ha presentado aquí, la TGS es una corriente teórica dentro de las ciencias sociales emparentada a la racionalidad formal-abstracta, que posee 
dos trazos imprescindibles: por un lado, la tendencia a buscar leyes generales que rompen con el estudio de cualquier tipo de particularidad "entre los sistemas" y, por otro lado, la cuestión del equilibrio y la tendencia a la obtención de una armonía y estabilidad dentro de todo sistema, sea este natural o de carácter social.

Estas determinaciones generales de la TGS aparece con suma claridad como una de las tendencias teórica en el debate contemporáneo del Trabajo Social argentino, sobretodo, en un corpus teórico que propone como finalidad de la intervención profesional la búsqueda del equilibrio de las relaciones sociales. Se ha analizado, como esto conduce a un excesivo énfasis en la familia y en lo relacional, junto a la "inducción comportamental" y la psicologización de la "cuestión social", y en la que se abandona cualquier perspectiva que apunte a efectivizar los derechos sociales, ya que las distintas problemáticas sociales, derivadas de la "cuestión social", deben ser resultas por los propios individuos y por la propia familia. Estos aspectos, junto al carácter "independiente" del Trabajo Social, expresan una nueva expresión del Trabajo Social tradicional.

Esta tendencia, junto a otra de carácter irracionalista y posmoderna existentes en el Trabajo Social argentino, ponen en riesgo la construcción de un Trabajo Social crítico capaz de develar las determinaciones presentes en la realidad y, a partir de ello, proponer estrategias de intervención que superen la inmediatez y la demanda institucional.

Recebido em 26/9/2014 - Aprovado em 24/11/2014

\section{Referencias bibliograficas}

BARROCO, M. L. Ética e Serviço Social: fundamentos ontológicos. São Paulo: Cortez, 2004.

BERTALANFFY, L. Von. Teoría general de los sistemas. México: Fondo de Cultura Económica, 1980. 
CALVO, L. Familia, resiliencia y red social: un abordaje experiencial en el Trabajo Social con familias. Buenos Aires: Espacio, 2009.

CHADI, M. Integración del Servicio Social y el enfoque sistémico-relacional. Buenos Aires: Espacio, 1997.

CHADI, M. Redes social en el Trabajo Social. Buenos Aires: Espacio, 2000.

. Familia y tratamiento familiar. Un desarrollo técnico-práctico. Buenos Aires: Espacio, 2005.

COUTINHO, C. N. El estructuralismo y la miseria de la razón. México: Editorial Era, 1973.

DURKHEIM, E. La división del trabajo social. Madrid: Ediciones Akal, 1987.

2002.

. Las reglas del método sociológico. Buenos Aires: Fondo de Cultura Económica,

GIANNA, S. Tendencias en el debate contemporáneo del trabajo social argentino: la influencia del racionalismo formal-abstracto y del irracionalismo en las concepciones teórico-metodológicas sobre la intervención profesional. Tesis (Doctorado) — Facultad de Ciencias Sociales, Universidad de Buenos Aires, Buenos Aires, 2014.

GOULDNER, A. La crisis de la sociología occidental. Buenos Aires: Amorrortu Editores, 1973.

LÖWY, M. ¿Qué es la sociología del conocimiento? México: Fontamara, 1991.

LUKÁCS, G. El asalto a la razón. La trayectoria del irracionalismo desde Schelling hasta Hitler. México: Fondo de Cultura Económica, 1959.

. Estética. La peculiaridad de lo estético. 1. Cuestiones previas y de principio. México: Grijalbo, 1966.

. El joven Hegel y el problema de la sociedad capitalista. México: Grijalbo, 1970.

. La crisis de la filosofía burguesa. Buenos Aires: La Pléyade, 1975.

. Marx y el problema de la decadencia ideológica. México: Siglo XXI, 1981.

. Para uma ontologia do ser social I. São Paulo: Boitempo, 2012.

MALLARDI, M. Cuestión social e individuo: aproximación a las tendencias contemporáneas de intervención sobre los problemas sociales. Escenarios, n. 19, p. 41-49, 2013. 
MARX, K. Crítica de la filosofia del derecho de Hegel. Buenos Aires: Ediciones Nuevas, 1965.

MÉSZÁROS, I. Estructura social y formas de conciencia. I. La determinación social del método. Caracas: Monte Ávila, 2011.

MONTAÑO, C. La naturaleza del Servicio Social: un ensayo sobre su génesis, su especificidad y su reproducción. São Paulo: Cortez, 1998.

NETTO, J. P. Capitalismo monopolista y servicio social. São Paulo: Cortez, 1997.

PARSONS, T. El sistema social. Madrid: Alianza, 1999.

REYNOSO, L.; CALVO, L. Trabajo Social y enfoque gestáltico. Buenos Aires: Espacio, 2003.

ROBERTSON, R.; PARSON, Talcott; RAISON, T. Los padres fundadores de la ciencia social. Barcelona: Anagrama, 1970.

SANTOS DE SOUZA, Renato. O funcionalismo sistêmico nas teorias social e organizacional: evolução e crítica. Revista Eletrônica de Administração, Porto Alegre, v. 7, n. 1, 2001. Disponible en: <http//www. Read.ea.ufrgs.br/edicoes/pdf/artigo_258.pdf>. Acceso en: 10 ago. 2014. 